Manuelle Medizin 2018 · 56:206 https://doi.org/10.1007/s00337-0180393-6

(c) Springer Medizin Verlag GmbH, ein Teil von Springer Nature 2018

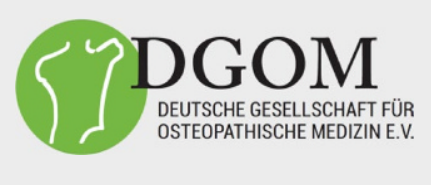

Deutsche Gesellschaft für Osteopathische Medizin (DGOM) e.V.

HGWR

Walter-Krause-Straße 11

68163 Mannheim

Tel.: 0621/4362 6692

Fax: 0621/4362 6691

Internet: kontakt@dgom.info

www.dgom.info
V.i.S.d.P.

Dr. med. Ute Beckmann

Berliner Str. 40

63654 Büdingen

\title{
Kursprogramm 2018 (Auszug)
}

\section{Grund- und Einführungskurs GKOMT \\ 02.-03.06.2018 \\ 15. -16.09 .2018$ \\ 16. -18.11 .2018$}

\section{MET 1}

07.-09.09.2018

Berlin

\section{MET 2}

25.-27.05.2018

Boppard - OV 5

\section{MFR 1}

25.-27.05.2018

Mannheim

\section{MFR 2}

06. -08.07 .2018$

Boppard - OV 6

\section{Counterstrain}

04.-06.05.2018

Mannheim

\section{Cranio 1}

25.-27.05.2018 Lübeck

\section{Klinischer Kurs}

20.-22.04.2018

Boppard

\section{Vis-Refresher}

04.-06.05.2018

Boppard

Die HGWR in Mannheim, die Hochschule für gesundheitsorientierte Wissenschaften Rheinneckar, nimmt zum 01.10.18 wieder neue Studenten der Osteopathischen Medizin auf.

Am 27.04.2018 findet ein Tag der offenen Tür statt.

Nähere Informationen finden Sie unter www.hgwr.de

\section{Masterkursreihe Osteopathische Pädiatrie}

Päd 1

30.11.-02.12.2018 Bochum J. Hohendahl / M.Riedel

\section{Masterkursreihe Osteopathische Geriatrie}

Manuelle Thermodiagnostik und Therapie
22.-24.06.2018
Mannheim
R. Wellstein

\section{Das Kiefergelenk (TMG)}

TMG1

20.-22.04.2018 Mannheim Hansen/Ewen

Osteopathie im biodynamischen Bereich (OBB)

Advanced Functional Methods

01.-03.06.2018 Athens/Ohio D. Eland

Funkt. Medizin nach Forte

\section{FMF 3}

19.-22.04.2018 Freiburg M. Forte

\section{Weitere Masterkurse}

Osteopathie und Ernährung

04.-06.05.2018 Mannheim R. Lebmeier/A. Galeazzi

\section{Dynamic Strain Vector Release}

06.-08.07.2018 Mannheim J. Hendryx

Advanced Muskelenergie-Techniken

13.-14.10.2018 Mainz

Das vollständige Kursprogramm ist einzusehen unter: www.dgom.info 